\title{
Energy Gaps and Kohn Anomalies in Elemental Superconductors
}

\author{
P. Aynajian, ${ }^{1}$ T. Keller, ${ }^{1,2}$ L. Boeri, ${ }^{1}$ \\ S. M. Shapiro, ${ }^{3}$ K. Habicht, ${ }^{4}$ B. Keimer ${ }^{1, *}$ \\ ${ }^{1}$ Max-Planck-Institute for Solid State Research, Heisenbergstr. 1, D-70569 Stuttgart, Germany \\ ${ }^{2}$ ZWE FRM-II, Technical University of Munich, Lichtenbergstr. 1, D-85748 Garching, Germany \\ ${ }^{3}$ Brookhaven National Laboratory, Upton, New York 11973-5000, USA \\ ${ }^{4}$ Hahn-Meitner-Institute, Glienicker Str. 100, D-14109 Berlin, Germany
}

*To whom correspondence should be addressed; E-mail: b.keimer@fkf.mpg.de.

The momentum and temperature dependence of the lifetimes of acoustic phonons in the elemental superconductors $\mathrm{Pb}$ and $\mathrm{Nb}$ was determined by resonant spin-echo spectroscopy with neutrons. In both elements, the superconducting energy gap extracted from these measurements was found to converge with sharp anomalies originating from Fermi-surface nesting (Kohn anomalies) at low temperatures. The results indicate electron many-body correlations beyond the standard theoretical framework for conventional superconductivity. A possible mechanism is the interplay between superconductivity and spin- or charge-density-wave fluctuations, which may induce dynamical nesting of the Fermi surface. 
Over the past half-century, a comprehensive framework based on the Bardeen-CooperSchrieffer formulation [1] has been developed for the interpretation of experimental data on superconductors. Although this framework has been challenged by the discovery of hightemperature superconductivity, it provides a remarkably successful description of the physical properties of conventional low-temperature superconductors [2]. Even today, however, the prediction of two of the most important quantities characterizing a superconductor - the transition temperature and the energy gap at the Fermi level - from first principles presents a formidable challenge to theory, because they depend exponentially on material-specific parameters such as the phononic and electronic densities of states and the electron-phonon coupling [3]. We present neutron scattering data on the lifetimes of acoustic phonons in $\mathrm{Pb}$ and $\mathrm{Nb}$ (the two elements with the highest superconducting transition temperatures, $T_{c}=7.2$ and $9.3 \mathrm{~K}$, respectively) that shed new light on the energy gap in conventional superconductors.

The energy gap can be directly determined in phonon lifetime measurements, because electron-phonon scattering is suppressed (and the phonon lifetimes are thus enhanced) for energies below the gap. Our data indicate a surprising relationship between the superconducting gap and the geometry of the Fermi surface, which also leaves an imprint on the phonon lifetimes [4]: For phonon wave vectors connecting nearly parallel segments of the Fermi surface, the electron-phonon scattering probability is enhanced, and lifetime extrema (termed Kohn anomalies) are generally expected. We have discovered hitherto unknown Kohn anomalies in both $\mathrm{Pb}$ and $\mathrm{Nb}$, and found that the low-temperature energy gap coincides with such an anomaly in both materials. This phenomenon has not been anticipated by the standard theoretical framework for conventional superconductors.

Both Kohn anomalies [5, 6, 7, 8] and superconductivity-induced phonon renormalization [9] have been observed by inelastic neutron scattering. However, as the requisite energy resolution is difficult to obtain, these investigations have been limited to a few selected materials, and both 
effects have thus far not been studied accurately in the same material. The systematic investigation reported here was made possible by recent advances in resonant spin-echo spectroscopy with neutrons [10, 11, 12], which have enabled the determination of the lifetimes of dispersive excitations with $\mu \mathrm{eV}$ energy resolution over the entire Brillouin zone. Briefly, the spin echo is generated on a triple-axis spectrometer by using radio-frequency magnetic fields to manipulate the spin polarization of neutrons scattered from a crystal before and after the scattering event. The excitation lifetime is then extracted from the spin-echo decay profile.

The measurements were taken on high-purity $\mathrm{Pb}$ and $\mathrm{Nb}$ single crystals. The resulting spinecho decay profiles for selected transverse acoustic phonons in $\mathrm{Pb}$ and $\mathrm{Nb}$ (Fig. 1) are well described by exponentials, corresponding to Lorentzian phonon spectral functions; deviations from Lorentzian lineshapes were not found within the experimental error. The spin-echo decay rate (proportional to the phonon linewidth and inversely proportional to its lifetime) decreases upon lowering the temperature, reflecting the loss of the electron-phonon decay channel in the superconducting state. The nonzero decay rate at the lowest temperatures is due to instrumental limitations, which can be quantitatively determined based on the phonon dispersion relations and the mosaic spreads of the single-crystal samples (see SOM [13]). The intrinsic Lorentzian phonon linewidths, $\Gamma$, are extracted by fitting the decay profiles to exponentials (lines in Fig. 1) and correcting for this instrumental contribution.

Fig. 2A shows the intrinsic wave vector dependent linewidths of the lowest-energy, transverse acoustic phonon mode $T_{1}$ of $\mathrm{Pb}$ along $q=(\xi, \xi, 0)$. At all temperatures, sharp anomalies in the phonon linewidths are seen at $\xi \sim 0.25, \sim 0.35$, and $\sim 0.50$ reciprocal lattice units (r.l.u.). Although the phonon spectrum of $\mathrm{Pb}$ has been studied extensively by conventional neutron spectroscopy, these particular features have not been recognized because of insufficient energy resolution. Fig. 2B shows that the same features also appear in the phonon dispersion relation: maxima in the phonon linewidth coincide with characteristic S-shaped deviations from 
the $q$-linear dispersion, as stipulated by the Kramers-Kronig relation that holds for all excitations in solids. Artefacts associated with the new measurement method would generally not be Kramers-Kronig consistent and can thus be ruled out. This implies that phonons with the anomalous wave vectors shown in Fig. 2A are intrinsically unstable towards decay into other elementary excitations. In principle, the decay products can be either other phonons (generated, for instance, by anharmonic terms in the lattice potential) or electron-hole pairs (originating from Kohn anomalies). The features at $\xi \sim 0.35$ and 0.5 can be associated with Kohn anomalies, because these wave vectors are known as nesting vectors of the Fermi surface [14]. Indeed, Kohn anomalies have been observed at these wave vectors in the longitudinal phonon branch of $\mathrm{Pb}[5,6]$, (see $\mathrm{SOM}[13])$. The origin of the feature at $\xi \sim 0.25$ is more subtle, because this wave vector does not match any known spanning vector of the Fermi surface. A possible origin is a three-phonon decay process previously observed in the spectrum of phonons in liquid helium, which are unstable because their phase velocity exceeds the velocity of sound [15, 16]. Indeed, accurate measurements of the phonon dispersions in $\mathrm{Pb}$ (Fig. 2B) show that the phonon phase velocity exceeds the sound velocity around $\xi \sim 0.25$, presumably as a consequence of the dispersion anomaly at $\xi \sim 0.35$. This process has thus far not been observed in solids and deserves further investigation. Anharmonic terms in the lattice potential may also contribute to the anomaly.

We will focus on the influence of superconductivity on the phonon linewidths below $T_{c}=$ $7.2 \mathrm{~K}$. As the superconductor is cooled below $T_{c}$, the electron-hole decay channel is closed (and $\Gamma$ is reduced) below the energy gap $2 \Delta(T)$. This effect is observed at low wave vectors $\xi$ in Fig. 2A. In particular, $\Gamma \rightarrow 0$ for $T<<T_{c}$ around $\xi=0.32$ (corresponding to a phonon energy of $2.47 \mathrm{meV}$, below the low-temperature limit of $2 \Delta \sim 2.7 \mathrm{meV}$ known from tunnelling measurements [19]). For lower energies around $\xi \sim 0.25$, however, $\Gamma$ remains nonzero even at the lowest temperatures, supporting the notion that the linewidth anomaly at this wave vec- 
tor originates from the three-phonon down-conversion process discussed above and/or lattice anharmonicity, and not from electron-hole pair production. We have removed the contribution of this process for clarity and show only the phonon linewidth $\Gamma_{e-p}$ directly attributable to the electron-phonon interaction (Fig. 3). As expected, $\Gamma_{e-p}$ exhibits a maximum due to the pileup of electronic density of states above $2 \Delta(T)$, which moves to progressively higher energies upon cooling and closely tracks the energy gap determined in prior tunnelling measurements [19] (inset in Fig. 3). Surprisingly, however, the superconductivity-induced maximum of $\Gamma_{e-p}$ merges with the Kohn anomaly as $T \rightarrow 0$. At $T=0.5 \mathrm{~K}$, both anomalies are indistinguishable within the measurement error.

In order to explore whether the coincidence of $2 \Delta(T \rightarrow 0)$ and the Kohn anomaly in $\mathrm{Pb}$ is accidental, we have performed similar experiments on phonons in $\mathrm{Nb}$, an elemental superconductor with a different Fermi surface and phonon spectrum. Fig. 4A shows the momentumdependent linewidths of the transverse acoustic phonon branch along $(\xi, 0,0)$ in $\mathrm{Nb}$ at temperatures above and below $T_{c}=9.3 \mathrm{~K}$. The data above $T_{c}$ are in fair agreement with prior work [9], but they reveal several sharp features that have not been been identified before. Based in part on ab-initio lattice dynamical calculations, they can be identified as Kohn anomalies (see below). The existence of a Kohn anomaly at $\xi \sim 0.17$ persisting up to room temperature has been suggested based on prior experimental work [7, 20]. As described above for $\mathrm{Pb}$, the linewidths are reduced below and enhanced above the gap for quasiparticle-pair production, $2 \Delta(T)$, in the superconducting state, and the low-temperature electron-phonon linewidth shows the expected dependence on wave vector (or energy). Similar to the observation in $\mathrm{Pb}$, the $2 \Delta(T \rightarrow 0)$ extracted from the low-temperature $\Gamma_{e-p}$ of $\mathrm{Nb}$ again coincides with the lowest-energy Kohn anomaly within the experimental error (Fig. 4B).

To help interpret these observations, we have calculated the phonon dispersions and linewidths in the framework of ab-initio density functional perturbation theory in the local-density approx- 
imation (LDA) (see SOM [13]), on a very fine mesh of q-points in reciprocal space. The phonon frequencies were obtained by diagonalization of the dynamical matrices, and the electronphonon linewidths by Allen's formula [21]. The results are in reasonable overall agreement with the experimental data (Figs. 3 and 4). In particular, both the phonon frequencies and the linewidths associated with Kohn anomalies in the high-energy transverse acoustic phonons of $\mathrm{Nb}$ (Fig. 4A) and in the longitudinal phonon of $\mathrm{Pb}$ (see $\mathrm{SOM}[13]$ ) are well described, indicating that the resolution of the calculations is sufficient to reproduce subtle structures in q-space.

The lowest-lying Kohn anomalies in the transverse-acoustic phonon branches of both $\mathrm{Pb}$ and $\mathrm{Nb}$ are, however, not reproduced by the calculations (Figs. 3A and 4A,B). These anomalies therefore originate in factors not included in the calculations, such as the relativistic spin-orbit coupling, phonon non-adiabaticity [22], or many-body correlations beyond Allen's formula [23, 24] or the LDA. As the Kohn anomalies in $\mathrm{Pb}$ and $\mathrm{Nb}$ are of comparable strength, the spin-orbit coupling (which is much stronger in $\mathrm{Pb}$ than in $\mathrm{Nb}$ ) cannot be responsible. Because of the large Fermi energies of both materials, non-adiabatic electron-phonon coupling effects should also be extremely weak.

This leaves electron correlation effects beyond the LDA as the most likely mechanism responsible for the low-energy Kohn anomalies. It seems reasonable to assume that the same correlations are also responsible for the observed coincidence of $2 \Delta(T \rightarrow 0)$ with the same anomalies. As the anomalies persist to temperatures above $100 \mathrm{~K}$, superconducting fluctuations are unlikely to be directly responsible. We note, however, that the formation of spin or charge density waves driven by electron correlations has been predicted for $\mathrm{Pb}$ and other elemental metals [25]. Although extensive searches for static density waves in simple metals have been unsuccessful, it is conceivable that fluctuations characteristic of such states dynamically enhance the nesting properties of the Fermi surface, and hence the propensity for Kohn anomalies in the phonon spectrum. Indeed, experiments on charge-density-wave materials such as $\mathrm{NbSe}_{2}$ 
have revealed Kohn anomalies [8] and Fermi-surface "pseudogaps" [26, 27] in the extended fluctuation regime at temperatures well above the onset of static density-wave order. Detailed theoretical work is required to assess whether interference between density-wave and superconducting correlations can limit the growth of the superconducting gap and lead to the observed convergence of both energy scales at low temperatures.

Our experiments on two different elemental superconductors demonstrate that the lowtemperature limit of the superconducting energy gap coincides with low-lying Kohn anomalies in transverse acoustic phonons. As both superconductors exhibit different lattice structures, phonon spectra, Fermi surfaces, and superconducting gaps, this coincidence cannot be accidental. While its origin is presently unclear, a specific scenario to explore in future theoretical work is the interplay between density-wave and superconducting correlations. It is interesting to point out a possible analogy to research on high-temperature superconductors, where an anomalous coincidence of the superconducting gap with a weakly temperature-dependent "pseudogap" has recently been reported in some regions of momentum space [28, 29, 30].

\section{References and Notes}

[1] J. Bardeen, L. N. Cooper, J. R. Schrieffer, Phys. Rev. 108, 1175 (1957).

[2] For a review, see J.R. Schrieffer, Theory of Superconductivity (Perseus Books, New York, 1999).

[3] For recent advances in ab-initio computation of superconducting properties, see M.A.L. Marques et al., Phys. Rev. B 72, 024546 (2005).

[4] W. Kohn, Phys. Rev. Lett. 2, 393 (1959).

[5] B. N. Brockhouse, T. Arase, G. Caglioti, A.D.B. Woods, Phys. Rev. 128, 1099 (1962). 
[6] R. Stedman, L. Almquist, G. Nilsson, G. Raunio, Phys. Rev. 163, 567 (1967).

[7] Y. Nakagawa, A. D. B. Woods, Phys. Rev. Lett. 11, 271 (1963).

[8] D.E. Moncton, J.D. Axe, F.J. DiSalvo, Phys. Rev. B 16, 801 (1977).

[9] S.M. Shapiro, G. Shirane, J.D. Axe, Phys. Rev. B 12, 4899 (1975).

[10] S.P. Bayrakci, T. Keller, K. Habicht, B. Keimer, Science 312, 1926 (2006).

[11] T. Keller et al., Phys. Rev. Lett. 96, 225501 (2006).

[12] T. Keller et al., Appl. Phys. A 74, S332 (2002).

[13] See supporting material on Science Online.

[14] J.R. Anderson, A.V. Gould, Phys. Rev. 139, A1459 (1963).

[15] H.J. Maris, W.E. Massey, Phys. Rev. Lett. 25, 220 (1970).

[16] L. P. Pitayevski, Y. B. Levinson, Phys. Rev. B 14, 263 (1976).

[17] D. L. Waldorf, Bull. Am. Phys. Soc. 5, 170 (1960).

[18] R. C. Dynes, V. Narayanamurti, J. P. Garno, Phys. Rev. Lett. 41, 1509 (1978).

[19] R.F. Gasparovic, B.N. Taylor, R.K. Eck, Sol. State Comm. 4, 59 (1966).

[20] M. A. Moore, D. I. Paul, Sol. State Comm. 9, 1303 (1971).

[21] P.B. Allen, Phys. Rev. B 6, 2577 (1972).

[22] S. Pisana et al., Nature Mat. 6, 198 (2007).

[23] E. Cappelluti, Phys. Rev. B 73, 140505 (2006). 
[24] O.V. Dolgov, O.K. Andersen, I.I. Mazin, Phys. Rev. B 77, 014517 (2008).

[25] A.W. Overhauser, Phys. Rev. 128, 1437 (1962).

[26] S.V. Borisenko et al., preprint available at www.arXiv.org/abs/0704.1544.

[27] T. Kiss et al., Nature Phys. 3, 721 (2007).

[28] K. Tanaka et al., Science 314, 1910 (2006).

[29] K. Terashima et al., Phys. Rev. Lett. 99, 017003 (2007).

[30] W.S. Lee et al., Nature 450, 81 (2007).

[31] We would like to thank O.K. Andersen, S.P. Bayrakci, S.V. Borisenko, M. Cardona, O. Dolgov, E.K.U. Gross, and G. Khaliullin for fruitful discussions, C.T. Lin for the Pb crystals, J. Mayor for the Nb crystals, and K. Buchner for technical assistance. 
Fig. 1. Spin-echo decay profiles of transverse acoustic phonons at $q=(0.26,0.26,0), E=2.32$ $\mathrm{meV}$, in $\mathrm{Pb}$ (upper two curves), and $q=(0.11,0,0), E=2.06 \mathrm{meV}$, in $\mathrm{Nb}$ (lower two curves) at selected temperatures. The spin polarization of the beam at the detector is plotted versus the spin-echo time $\tau$ [10, 11]. The lines are the results of fits of exponentials (corresponding to Lorentzian spectral functions) to the data. The inset shows a conventional triple-axis scan through the phonon in $\mathrm{Pb}$.

Fig. 2. (A) Linewidths of transverse acoustic phonons along $q=(\xi, \xi, 0)$ in $\mathrm{Pb}$ at selected temperatures. The data were obtained by correcting the measured spin-echo decay rates for instrumental effects (see SOM [13]). The grey symbols are the results of ab-initio lattice-dynamical calculations, as described in the text. (B) Dispersion relation of the same phonon extracted from triple-axis data. The inset shows the phonon phase velocity $(E / q)$ computed from the data. The blue line in the main panel and the black line in the inset represent the experimentally determined sound velocity [17].

Fig. 3. Contribution of the electron-phonon interaction to the linewidth of the transverse acoustic phonon along $q=(\xi, \xi, 0)$ in $\mathrm{Pb}$. The corresponding phonon energy $E$ is provided by the scale at the top; note the nonlinear $E$-versus- $q$ relationship (Fig. 2B). The lines are results of least-squares fits to the Bardeen-Cooper-Schrieffer (BCS) excitation spectrum function [18]. The inset shows the temperature dependence of the superconducting energy gap (filled squares) and Kohn anomaly (open triangles) extracted from the fits. The line in the inset shows the BCS expression for the superconducting gap [2], which was experimentally confirmed by tunnelling spectroscopy [19].

Fig. 4. (A) Linewidths of transverse acoustic phonons along $q=(\xi, 0,0)$ in $\mathrm{Nb}$ at two different temperatures. The grey symbols are the results of lattice-dynamical calculations, as described in the text. (B) Blowup of the low- $q$ segment of panel A. The corresponding phonon energy $E$ is provided by the scale at the top. The lines are guides-to-the-eye. 


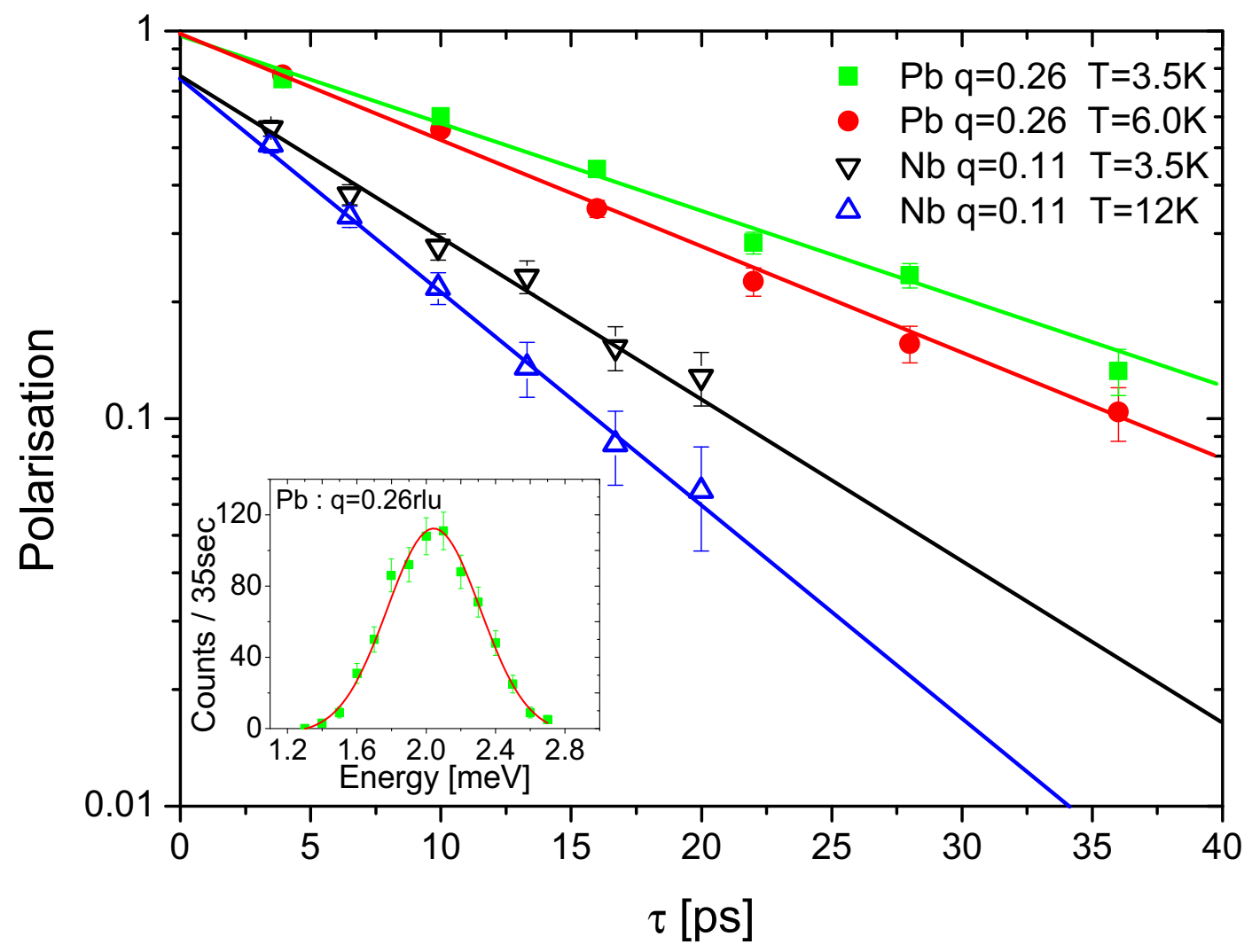

11

Figure 1: 

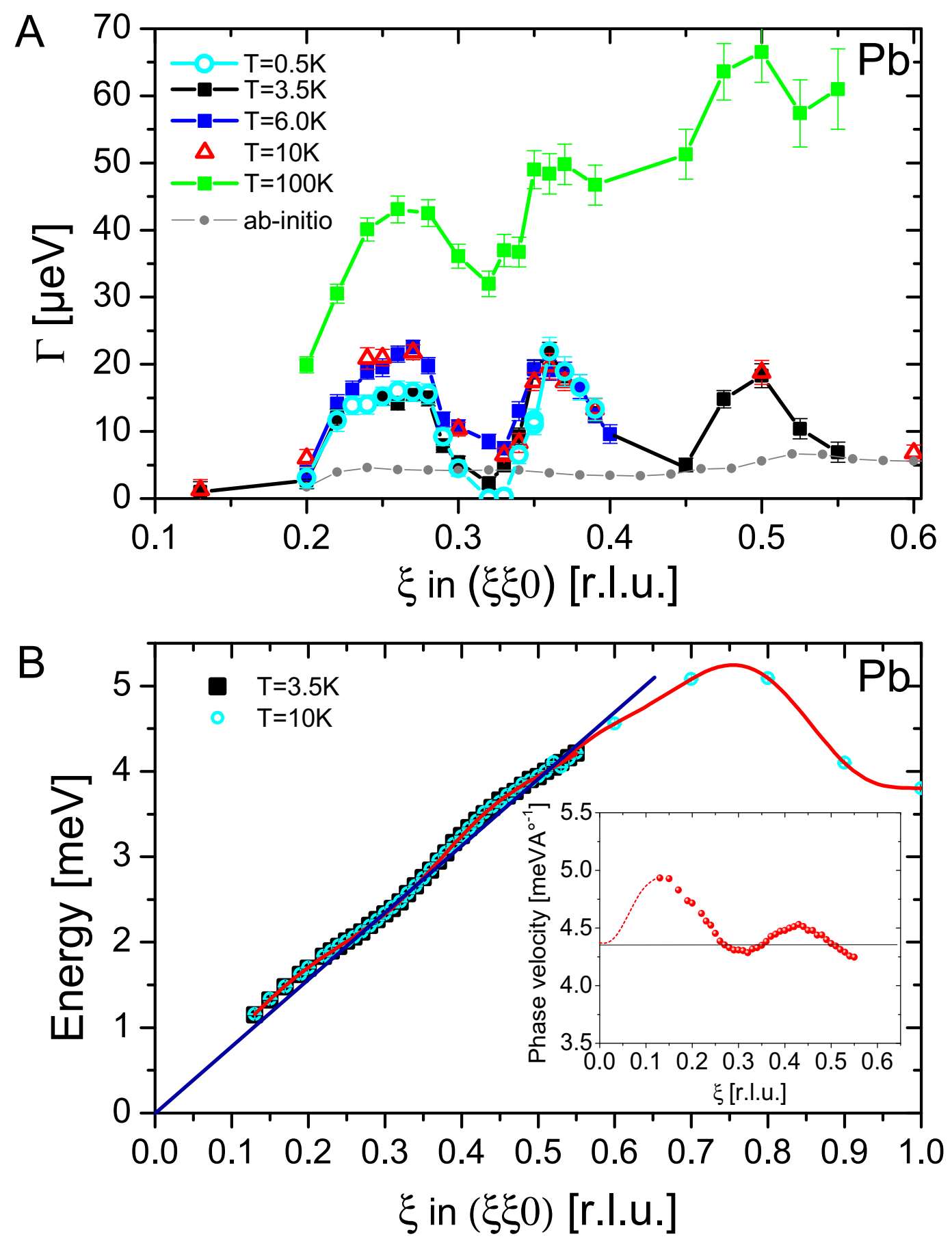


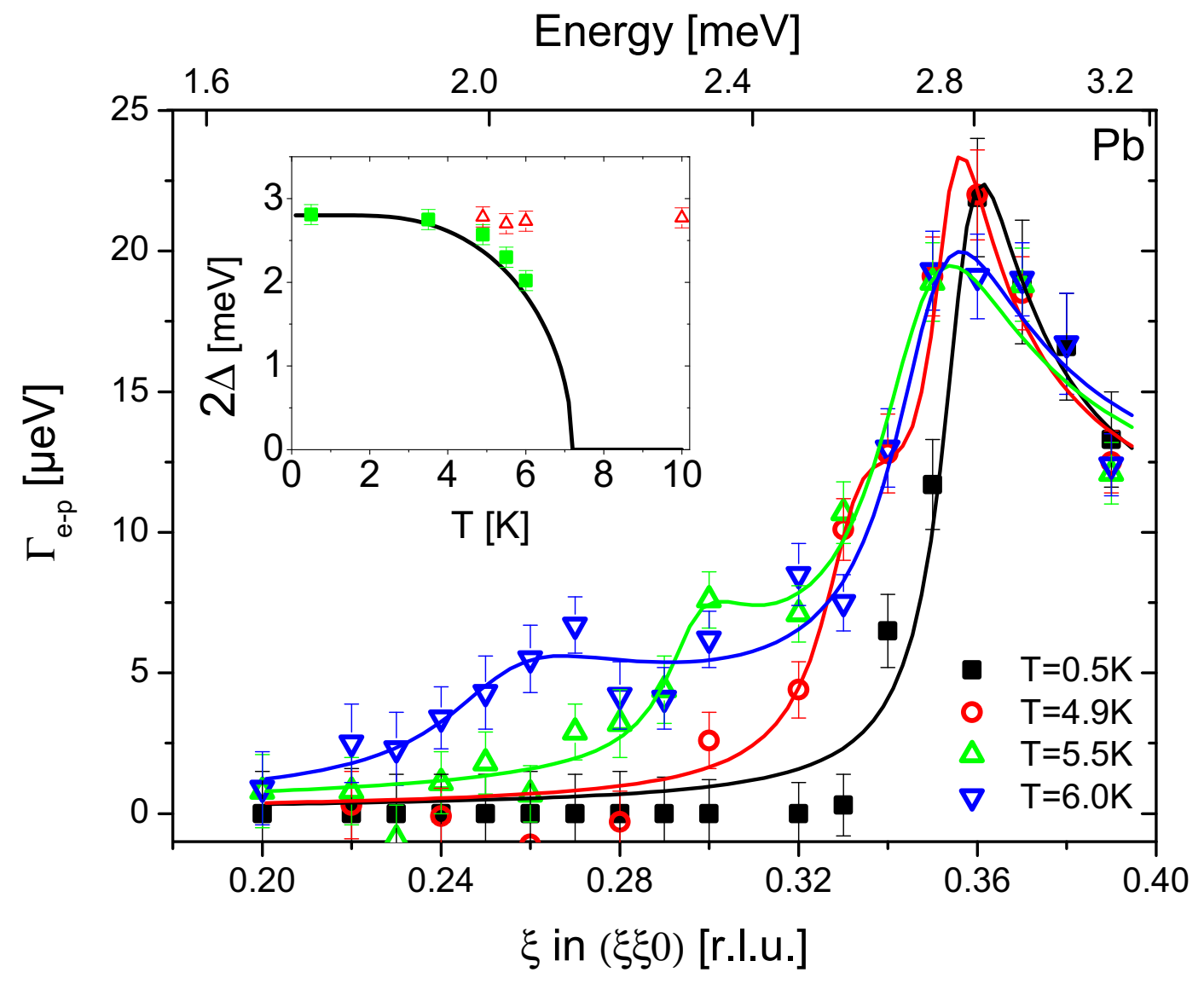



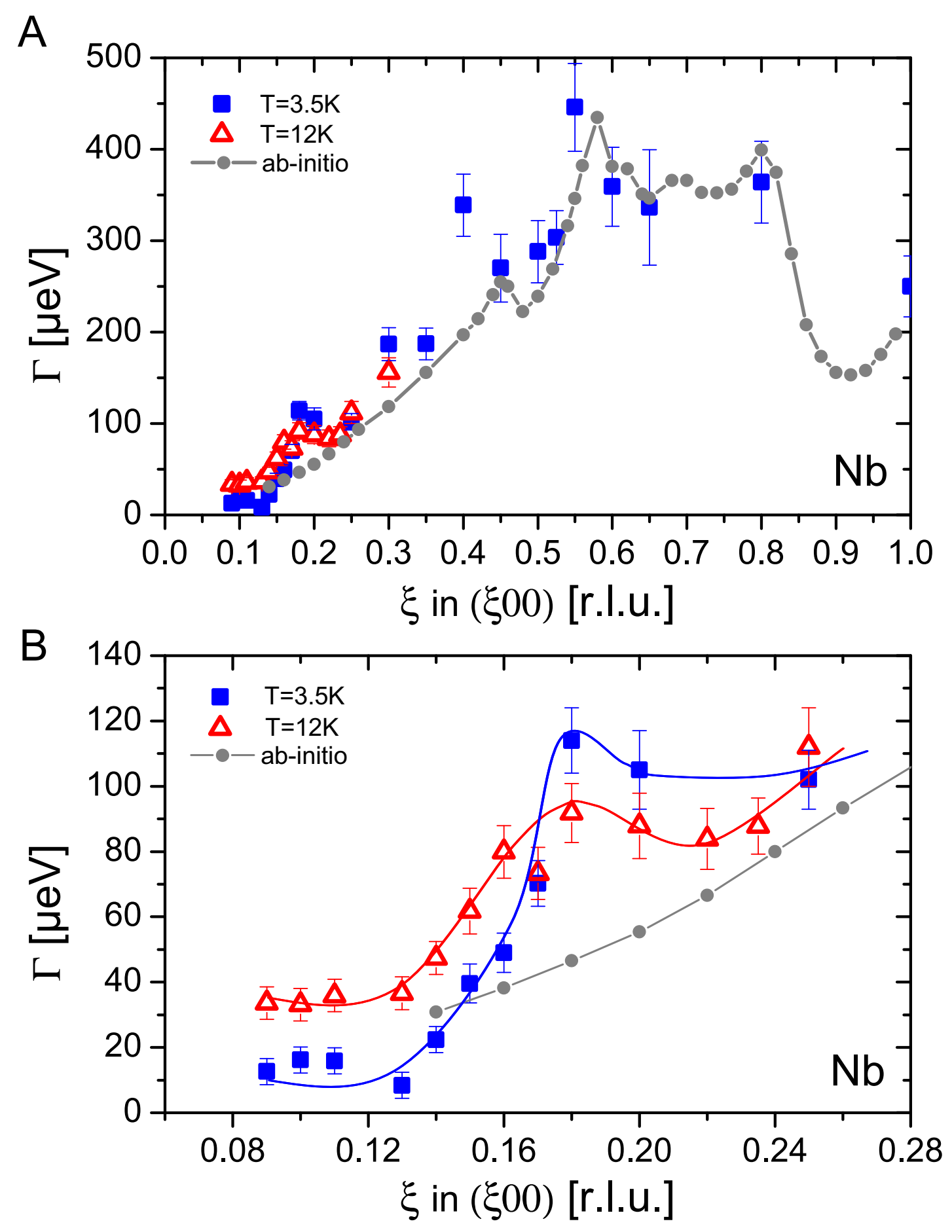